\title{
Children's higher order cognitive abilities and the development of secondary memory
}

\author{
Duneesha De Alwis and Joel Myerson \\ Washington University, St. Louis, Missouri \\ TAMARA Hershey \\ Washington University School of Medicine, St. Louis, Missouri \\ AND \\ SANDRa HaLe \\ Washington University, St. Louis, Missouri
}

\begin{abstract}
The relations between higher cognitive abilities and immediate and delayed recall were studied in 57 children (6-16 years of age). The participants were tested repeatedly on free recall of a supraspan list (Children's Memory Scale), and their fluid ability was also assessed (Woodcock-Johnson III Spatial Relations). Consistent with Unsworth and Engle's (2007) account of the relation between memory and higher order cognition, the children's fluid ability was significantly correlated with retrieval from secondary memory, regardless of whether it was measured using immediate or delayed recall. Multiple regression analyses provided further support for this view, revealing that measures of immediate and delayed retrieval from secondary memory accounted for the same variance in the children's fluid ability.
\end{abstract}

Children's memory and higher order cognitive abilities both improve with age, but the nature of the relation between these two developmental trends is not yet well understood. Much recent research on higher order cognitive abilities in adults has been focused on working memory's contribution to these abilities, and developmental researchers have also pursued this approach (e.g., Bayliss, Jarrold, Baddeley, \& Gunn, 2005; Kail, 2007). Working memory tasks (e.g., reading span, operation span), sometimes termed complex span tasks, require processing, as well as short-term storage of information. This processing requirement distinguishes complex span tasks from simple span tasks, which require only storage. Complex span tasks have been reported to be better predictors of higher order abilities than are simple span tasks (e.g., Engle, Tuholski, Laughlin, \& Conway, 1999; Swanson \& Howell, 2001). However, recent research with both children (e.g., Bayliss et al., 2005) and adults (e.g., Colom, Shih, Flores-Mendoza, \& Quiroga, 2006) has raised questions regarding this distinction.

Unsworth and Engle (2007) recently proposed a twocomponent theory that takes a new approach to this issue. According to Unsworth and Engle (2007), both simple and complex span tasks involve maintaining items in primary memory and retrieving them from secondary memory, but they differ in the relative contributions made by these two processes. They suggest that retrieval from secondary memory becomes important to working memory perfor- mance under two conditions: (1) when processing that is interleaved between the encoding of successive memory items displaces those items from primary memory (as on complex span tasks) and (2) when the number of items exceeds the capacity of primary memory, even if the task involves only storage (as in recall of supraspan lists). Although both primary and secondary memory are hypothesized to play a role in the correlation between working memory and higher order cognition, Unsworth and Engle (2007) presented data showing that primary and secondary memory abilities are largely independent of each other and that the unique contribution of secondary memory to higher order cognition is roughly twice that of primary memory.

If Unsworth and Engle (2007) are correct, developmental and individual differences in children's higher order cognitive abilities should be more strongly related to their secondary memory than to their primary memory abilities. In the present study, we tested this hypothesis. To measure children's primary and secondary memory, we made use of the long-standing idea (reiterated by Unsworth and Engle, 2007) that in immediate free recall, items from the beginning of a supraspan list are retrieved from secondary memory, whereas items from the end tend to be retrieved from primary memory (Waugh \& Norman, 1965; for recent neuroimaging evidence, see Talmi, Grady, Goshen-Gottstein, \& Moscovitch, 2005). Unsworth and Engle (2007) also assumed that the capacity of primary

D. De Alwis, dsranatu@wustl.edu, or J. Myerson, jmyerson@wustl.edu 
memory is approximately four items (Cowan, 2001) and that secondary memory involvement in simple storage tasks depends on the extent to which list length exceeds that number. In the present study, we tested these assumptions and predictions of Unsworth and Engle's (2007) twocomponent model.

According to Unsworth and Engle (2007), the first 10 (prerecency) items of 14-item lists, like those used in the present study, should be in secondary memory and thus vulnerable to proactive interference, whereas the last 4 items should not. Some researchers have questioned whether recall of the end of a list depends on a simple readout from primary memory (e.g., Bhatarah, Ward, \& Tan, 2006). Therefore, in the present study, we examined the effects of proactive interference in order to assess the validity of using immediate recall of recency items as a measure of primary memory and immediate recall of prerecency items as a secondary memory measure. Additional measures of secondary memory used in the present study relied on the idea, also of long standing, that items recalled after practice and after a filled delay are retrieved from secondary memory. If Unsworth and Engle's (2007) two-component theory is correct, immediate recall of prerecency items should predict higher order abilities better than should immediate recall of recency items, and most of the variance in higher order abilities accounted for by immediate and delayed retrieval from secondary memory should be shared.

\section{METHOD}

\section{Participants}

All of the participants (29 male and 28 female, ranging in age from 6 to 16 years, $M=11.69$ years, $S D=3.06$ ) were enrolled in a longitudinal study on diabetes in children (for the recruitment procedures, see Perantie et al., 2007). The data reported here are from the normally developing, nondiabetic siblings of diabetic children in the longitudinal study.

\section{Stimuli and Procedure}

The participants were tested individually on a battery of cognitive, motor, and reasoning tasks. For this study, we focused on two of these tasks: the Word Lists test from the Children's Memory Scale (Cohen, 1997) and the Spatial Relations test from WoodcockJohnson III (Woodcock, McGrew, \& Mather, 2001).

Word Lists (Children's Memory Scale). The Word Lists test is used to assess verbal short-term and long-term memory and verbal learning in children (Cohen, 1997). On Trial 1, the experimenter read a list of 14 unrelated words at approximately one item per second, and the child taking the test tried to recall as many items as possible. The experimenter then repeated the items that had been missed, and the child again tried to recall the entire study list (Trial 2). Selective reminding of missed items was also provided prior to Trials 3 and 4, after which the child heard and then tried to recall a new 14word distractor list (Trial D). Next, the child was asked to recall the original study list (Trial 5). Following a 30-min delay, during which other cognitive tasks were performed, the child was asked to recall the study list one more time (Trial 6).

For the most part, the test was administered as was described in the test manual (Cohen, 1997). The test consists of two supraspan lists, one of which is usually the study list, whereas the other is the distractor list, but in the present study, the lists were counterbalanced across participants. Following the standard procedure, the experimenter recorded whether each item was correctly recalled but did not record the order of recall. Therefore, it was not possible to apply the Tulving and Colotla (1970) rule for distinguishing retrieval from primary and secondary memory, and the effects of proactive interference were examined to validate the division into primary and secondary memory components. Although probed recall (e.g., Cantor, Engle, \& Hamilton, 1991) may avoid the issue of recall order (Tan \& Ward, 2000), the Word Lists test has the advantage of being a standardized instrument with good reliability and demonstrated validity (Cohen, 1997).

Spatial Relations (Woodcock-Johnson III). This untimed test is used to assess visuospatial thinking and spatial intelligence (Schrank, McGrew, \& Woodcock, 2001). On each trial, the child was shown a target shape and six pieces and asked to choose those pieces that would make the target shape. The task consisted of 33 test trials, with 18 trials that required the choice of two pieces and 15 trials that required three pieces to complete the shape.

The Spatial Relations test is sometimes described as measuring visuospatial thinking, or Gv (Schrank et al., 2001), but it has been shown to tap fluid ability as well (McGrew \& Flanagan, 1998). Although the Spatial Relations test places a high demand on visuospatial abilities, such as mental rotation, it also places a high demand on working memory, an ability associated with fluid intelligence (Engle et al., 1999; Kane \& Engle, 2002). According to Schatz and Roberts (2005), the Woodcock-Johnson Spatial Relations test provides a good measure of children's fluid ability because it works well with a wider age range than do alternative measures in the Woodcock-Johnson battery.

\section{RESULTS}

The recall data from each trial of the Word Lists test were divided into three sections: beginning (first 4 items), middle (next 6 items), and end (last 4 items). Successful recall of the first 10 items immediately following the initial presentation of a list was hypothesized to involve retrieval from secondary memory, whereas the last 4 items were hypothesized to be unloaded directly from primary memory. As was expected, immediate recall on Trial 1 and Trial D showed clear serial position effects, with children

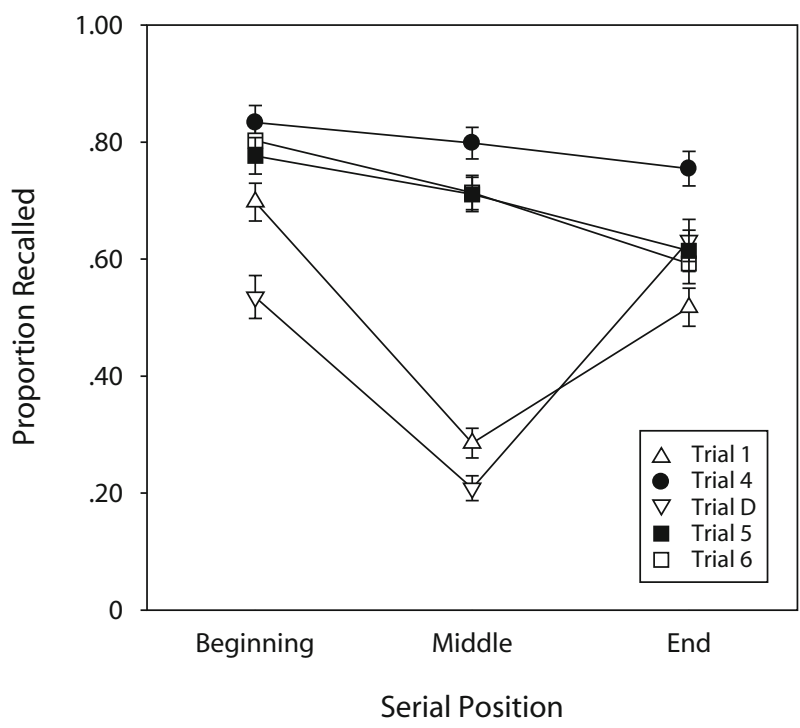

Figure 1. Proportion of items from each section (beginning, middle, and end) of the list that were accurately recalled on Trials 1, 4, D, 5, and 6. Error bars indicate the standard errors of the means. 
Table 1

Characteristics of the Age Groups in Figure 2

\begin{tabular}{llcc}
\hline \multicolumn{1}{c}{ Group } & $N$ & Mean Age & $S D$ \\
\hline 6-9 years & 17 & 7.79 & 1.24 \\
10-12 years & 23 & 12.08 & 0.74 \\
13-16 years & 17 & 15.16 & 1.18 \\
\hline
\end{tabular}

recalling more items from the beginning and end of the list and fewer items from the middle (see Figure 1). In contrast, there was little evidence of serial position effects in the recall data from Trials 4-6. This was expected on the basis of the assumption that repeated practice would result in items from all portions of the list being learned and encoded in secondary memory. As can be seen in Figure 1, by Trial 4 , not only had the retrieval of items from the beginning of the list improved from Trial 1 levels $[t(56)=$ $3.83, p<.001]$, but the recall of items from the end of the list had also improved significantly with practice $[t(56)=$ $5.95, p<.001]$. By Trials 5 and 6 , both of which followed filled delays, all of the items would obviously have to be retrieved from secondary memory.

Consistent with the results of previous studies (e.g., Cole, Frankel, \& Sharp, 1971; Spitzer, 1976), the immediate recall of items from the beginning of both the study and the distractor lists improved significantly with age (correlations of .43 and .54, respectively), whereas the immediate recall of items from the end of the lists did not (correlations with age of .16 and .00 , respectively). A repeated measures analysis using the GLM revealed a significant difference between the slope of the regression of recall of the beginning of the list on age and the slope of the regression for recall of the end of the list $[F(1,55)=8.83, p=$ $.004]$. These age trends can be seen in Figures 2A and 2B, in which (for purposes of illustration only) the sample was divided into three age groups (see Table 1).

Figures 2A and 2B also show that when recall of the first (study) list on Trial 1 was compared with recall of the second (distractor) list on Trial D, selective proactive interference with recall of the initial portion of the distractor list was observed. As can be seen, recall of the first four items (Figure 2A) was better on Trial 1 than on Trial $\mathrm{D}$ for all three age groups, whereas recall of the last four items (Figure 2B) was better on Trial D. This pattern of results was confirmed by a repeated measures analysis using the GLM, which revealed a significant interaction between portion of the list (beginning vs. end) and trial type (Trial 1 vs. Trial D) $[F(1,55)=4.19, p=.046]$ but no three-way interaction among portion of the list, trial type, and age $[F(1,55)=1.25, p=.268]$. The observed selective effect of proactive interference is consistent with the hypothesis that recall of the initial portion reflects retrieval from secondary memory, whereas the recency portion (last four items) largely reflects a readout from primary memory.

The hypothesis that retrieval from secondary memory is a significant predictor of fluid ability, regardless of whether recall is immediate or delayed, may be evaluated by examining the intercorrelations among recall on Trials 1, 4, 5, and 6 and the raw scores from the Spatial Rela- tions test. If the hypothesis is correct, two things should be observed. Recall from the beginning and middle portions of the list on Trial 1 and recall from Trials 4-6, all of which are hypothesized to involve retrieval from secondary memory, should be significantly correlated with each other and also with the Spatial Relations test scores. As can be seen in Table 2, the results showed the predicted pattern: The recall measures hypothesized to reflect retrieval from secondary memory all tended to correlate positively with each other and with the Spatial Relations scores, regardless of the timing of the recall test.

If a child recalled the items in serial order, of course, even the items from the end of the list might be retrieved from secondary memory. To the extent that this occurred, however, it would bias the results toward finding a positive correlation between immediate recall from the end of the list and delayed recall, which clearly depends on retrieval from secondary memory. However, none of the correla-

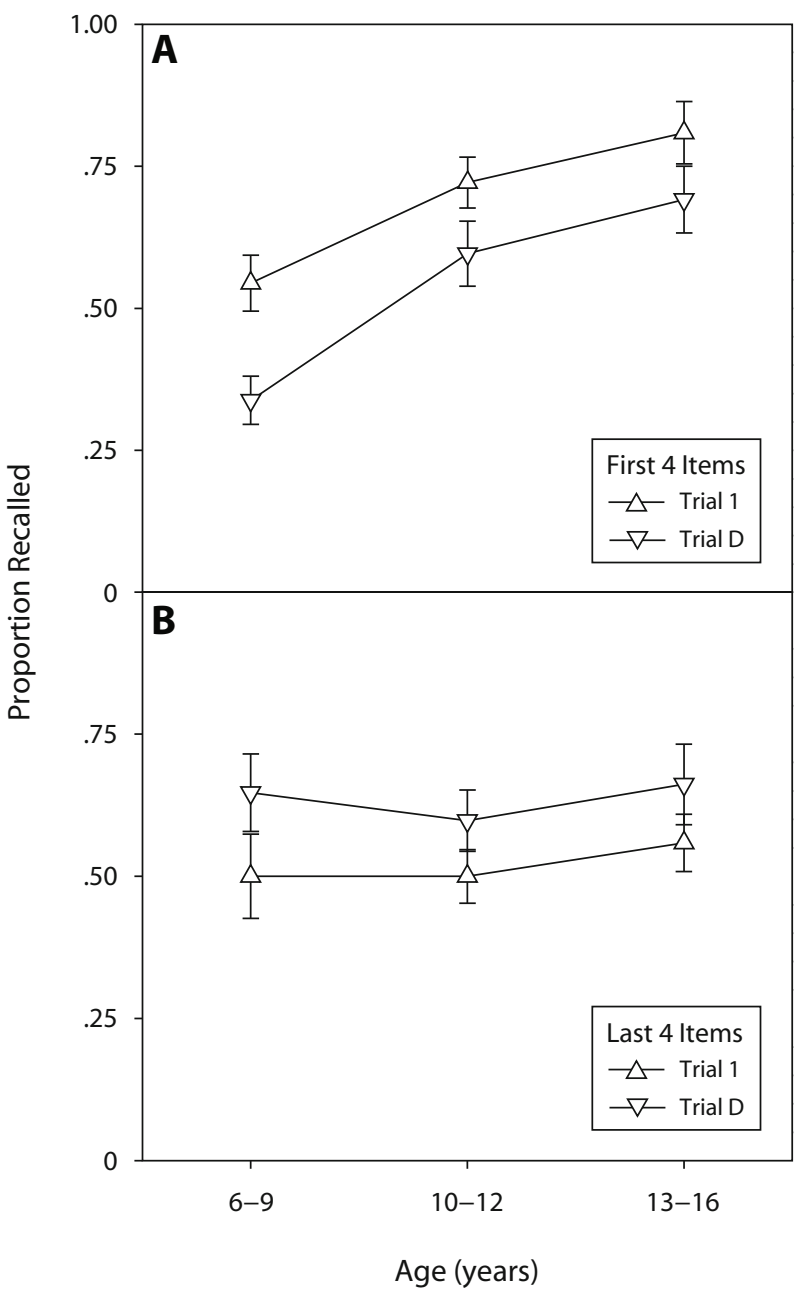

Figure 2. Proportion of items correctly recalled from the beginning (A) and end (B) of the list on Trial 1 and Trial $D$ as a function of age. For the two older groups, the number of items correctly recalled from the beginning of the list was significantly greater than the number of items correctly recalled from the end of the list. Error bars indicate the standard errors of the means. 
Table 2

Intercorrelations of Recall Measures and Fluid Ability

\begin{tabular}{lccccccc}
\hline & & \multicolumn{3}{c}{ Trial 1 } & & & \\
\cline { 3 - 5 } & Age & Beginning & Middle & End & Trial 4 & Trial 5 & Trial 6 \\
\hline Trial 1 & & & & & & & \\
$\quad$ Beginning & $.43^{*}$ & - & & & & & \\
$\quad$ Middle & .15 & .15 & - & & & & \\
$\quad$ End & .16 & -.13 & -.12 & - & & & \\
Trial 4 & $.57^{*}$ & $.39^{*}$ & $.34^{*}$ & .11 & - & & \\
Trial 5 & $.34^{*}$ & $.39^{*}$ & $.30^{*}$ & .06 & $.67^{*}$ & - & \\
Trial 6 & $.49^{*}$ & $.49^{*}$ & $.41^{*}$ & -.18 & $.74^{*}$ & $.80^{*}$ & - \\
Spatial Relations & $.68^{*}$ & $.38^{*}$ & $.30^{*}$ & -.07 & $.65^{*}$ & $.34^{*}$ & $.52^{*}$ \\
\hline${ }^{*} p<.05$. & & & & & &
\end{tabular}

tions between immediate recall of the end of the study list and recall after practice or after a delay were significant, whereas all of the correlations with immediate recall from the beginning of the list were significant (see Table 2). Moreover, a similar pattern of results was obtained for immediate recall of the distractor list: Recall of the beginning of the list, but not the end, correlated significantly with delayed recall on Trial 6 ( $r \mathrm{~s}=.35$ and .16 , respectively). Recall of the middle of the distractor list was not correlated with delayed recall $(r=.11)$, perhaps because of a restriction of range (one fourth of the participants recalled none of the middle items on the second list, as compared with one eighth for the middle of the first list, and most could recall no more than one of the six items).

Comparisons of the correlations involving immediate recall of the three sections of the study list on Trial 1 with correlations involving recall of the whole list on subsequent trials are complicated by the fact that different numbers of items contribute to the different memory measures. Accordingly, Table 3 presents correlations between the Spatial Relations test scores and recall of the first four and last four items of the list on Trials 1, 4, D, 5, and 6. The scores on the Spatial Relations test were correlated with the recall of the beginning of the list on all trials (although the correlation for Trial 5 was at the trend level), consistent with the hypothesis that fluid ability depends on retrieval from secondary memory, regardless of whether retrieval is assessed on the basis of immediate or delayed recall. The scores on the Spatial Relations test also were significantly correlated with recall of the end of the list on Trials 4, 5, and 6 ( $r$ s of .36, .30, and .36, respectively), consistent with the hypothesis that after repeated practice or after a filled delay, recall of the end of the list involved retrieval from secondary memory. In contrast, correlations with the end

Table 3

Correlations of Fluid Ability With Recall of the Beginning and End Sections of the Study and Distractor Lists

\begin{tabular}{ccc}
\hline Trial & First Four Items & Last Four Items \\
\hline 1 & $.38^{*}$ & -.07 \\
4 & $.52^{*}$ & $.36^{*}$ \\
$\mathrm{D}$ & $.45^{*}$ & .14 \\
5 & $.24^{\dagger}$ & $.30^{*}$ \\
6 & $.36^{*}$ & $.36^{*}$ \\
${ }^{*} p<.05 . \quad{ }^{\dagger} .05<p<.08$. &
\end{tabular}

of the list were not significant for trials involving immediate recall (Trial 1 and Trial D), consistent with the hypothesis that immediate recall of the end of the list involved primary rather than secondary memory.

In order to determine whether the observed pattern of results would generalize to individual differences among children, partial correlations (controlling for age) were also examined. If individual differences in retrieval from secondary memory predict individual differences in children's higher order cognitive abilities, the correlations between secondary memory measures and spatial relations scores should remain significant when age is statistically controlled. Because it was hypothesized that the immediate recall of the beginning and middle portions of the list both measure retrieval from secondary memory, and because the immediate recall of the beginning and of the middle sections were both adversely affected by proactive interference and showed similar patterns of correlation with other secondary memory measures, as well as with fluid ability (see Table 2), the immediate recall scores for the first two sections of the list were combined into one measure.

As can be seen in Table 4, the partial correlations showed the expected pattern, with the exception of recall of the study list on Trial 5 (perhaps because of retroactive interference from the distractor list). All of the other measures hypothesized to reflect retrieval from secondary memory were positively correlated with each other and with fluid ability, even after controlling for age. Notably, when the last 4 items were included in the measure of immediate recall on Trial 1 (so that it was based on recall of all 14 items, rather than on that of just the first 10), the correlations with delayed recall on Trial 6 and fluid ability (Spatial Relations) did not increase (partial correlations of

Table 4

Intercorrelations of Recall and Fluid Ability After Statistically Controlling for Age

\begin{tabular}{|c|c|c|c|c|}
\hline & $\begin{array}{c}\text { Trial } 1 \\
\text { (First } 10 \text { Items) }\end{array}$ & Trial 4 & Trial 5 & Trial 6 \\
\hline Trial 4 & $.33^{*}$ & & & \\
\hline Trial 5 & $.37^{*}$ & $.61^{*}$ & & \\
\hline Trial 6 & $.50^{*}$ & $.65^{*}$ & $.77^{*}$ & \\
\hline Spatial Relations & $.26^{\dagger}$ & $.43^{*}$ & .15 & $.30^{*}$ \\
\hline
\end{tabular}


.38 and .12 for immediate recall of all 14 items vs. .50 and .26 for the first 10 items), consistent with the hypothesis that recall of the last 4 items involves processes that are distinct from secondary memory and only weakly related to fluid ability. Taken together, the pattern of partial correlations is consistent with the hypothesis that the ability to retrieve information from secondary memory is a predictor of individual differences in higher order cognition in children.

In order to determine whether immediate and delayed recall tests tap the same ability (i.e., retrieval from secondary memory), one that is important for higher order cognition, multiple regression analyses were used to partial the variance in performances on the Spatial Relations test. When age was entered into the analysis, followed by recall of the first 10 items of Trial 1 , recall on Trial 1 accounted for a significant additional proportion of the variance $[F(1,54)=4.53, p=.038]$. Adding recall of the first 10 items of Trial 6 (to equate the level of aggregation) did not result in a further increase $[F(1,53)=1.42$, n.s.]. When the order of entry of the recall measures was reversed, Trial 6 accounted for a significant proportion of the variance $[F(1,54)=4.60, p=.037]$ over and above that explained by age, but adding Trial 1 did not result in a further increase $[F(1,53)=1.36$, n.s.]. Taken together, the results of these multiple regression analyses suggest that retrieval from secondary memory on immediate and delayed recall tests accounts for the same variance in children's fluid ability. A final multiple regression analysis revealed that when age was entered first, it accounted for $45.7 \%$ of the variance, but including recall of items from the end of the lists on Trial 1 and Trial D in the model did not result in a significant increase, suggesting that individual differences in retrieval from primary memory do not contribute to the explanation of the variance in children's fluid ability.

\section{DISCUSSION}

In the present study, we tested the hypothesis that retrieval from secondary memory is predictive of higher order cognitive abilities in children. The immediate recall of the first 10 (prerecency) items of a 14-word list was assumed to measure retrieval from secondary memory, and selective effects of proactive interference validated this interpretation. Consistent with Unsworth and Engle's (2007) two-component theory, children's immediate recall of prerecency items predicted both age and individual differences in performances on a test of fluid ability and shared the variance accounted for by delayed recall.

With regard to developmental trends in free recall, our results are consistent with previous studies of list learning in children. Although clear primacy and recency effects were observed at all ages in these studies, only the primacy effect increased as a function of age (Cole et al., 1971; Spitzer, 1976). One interpretation of these findings is that they reflect age-related differences in strategy, but these findings have also been interpreted as reflecting the fact that, regardless of strategy, children's secondary memory improves with age, whereas their primary memory does not. Consistent with the results of previous studies, we observed significant age-related increases in the immediate recall of items from the beginning of supraspan lists, as well as in recall after practice and in delayed recall, whereas the immediate recall of items from the end of the lists did not improve with age. In contrast, Cowan and his colleagues argued that working memory capacity, by which he appears to mean something very similar to primary memory, does improve with age (Cowan, Nugent, Elliott, Ponomarev, \& Saults, 1999; Cowan, Nugent, Elliott, \& Saults, 2000). Although the present results do not preclude this possibility, they do appear to pose problems for this view.

Our results also indicate that recall of items from the beginning of supraspan lists and recall of items from the end of such lists differ not only in their relations with age, but also in their relations with higher order cognitive abilities. The recall of items from the end of the supraspan lists, which reflected the readout from primary memory, was not significantly correlated with fluid ability, whereas the recall of items from the beginning of the list, which reflected the retrieval from secondary memory, was correlated. These results do not preclude a relation between primary memory and higher order abilities, but they are consistent with Unsworth and Engle's (2007) contention that this relation is not as strong as that between secondary memory and higher order abilities. Similar patterns of correlations between secondary memory measures and fluid ability were observed when age was statistically controlled and when it was not. This finding suggests that differences in retrieval from secondary memory may help explain both age and individual differences in children's higher order cognitive abilities.

Overall, the present findings are consistent with Unsworth and Engle's (2007) hypothesis that the ability to retrieve information from secondary memory predicts higher order cognitive abilities. Although the role that retrieval from secondary memory plays in some aspects of higher order cognition (e.g., reading comprehension) is obvious, its role in other higher order cognitive abilities may not be so apparent. Unsworth and Engle (2007) argued that the ability to retrieve items from secondary memory may be important for reasoning and problem solving, because partial solutions to a problem must often be displaced from primary memory in order to work on other parts of the problem, only to be retrieved from secondary memory later in order to construct the complete solution. Indeed, the ability to solve the problems on the Spatial Relations test clearly depends on the ability to accumulate partial solutions. Moreover, Unsworth and Engle's (2007) argument would appear to apply equally well regardless of the age of the individual problem solver and, thus, may well account for the relation between children's fluid ability and both individual and age differences in recall as observed in the present study. The reason that retrieval from secondary memory improves with age, however, is not directly addressed by this line of argument, and further developmental research on the relation between age dif- 
ferences in recall and higher order abilities will be needed to resolve this issue.

\section{AUTHOR NOTE}

This work was supported by NIDDK Grant DK64832 to T.H. We thank Dana Perantie, Audrey Lim, Jenny Wu, and Patrick Weaver for their help in data collection and Tassy Hayden for her help in data entry and coding. Correspondence concerning this article should be addressed to D. De Alwis or J. Myerson, Department of Psychology, Washington University, Campus Box 1125, St. Louis, MO 63130 (e-mail: dsranatu@ wustl.edu or jmyerson@wustl.edu).

\section{REFERENCES}

Bayliss, D. M., Jarrold, C., Baddeley, A. D., \& GunN, D. M. (2005). The relationship between short-term memory and working memory: Complex span made simple? Memory, 13, 414-421.

Bhatarah, P., Ward, G., \& TAN, L. (2006). Examining the relationship between free recall and immediate serial recall: The effect of concurrent task performance. Journal of Experimental Psychology: Learning, Memory, \& Cognition, 32, 215-229.

Cantor, J., Engle, R. W., \& Hamilton, G. (1991). Short-term memory, working memory, and verbal abilities: How do they relate? Intelligence, 15, 229-246.

Cohen, M. J. (1997). Children's Memory Scale. San Antonio: Psychological Corporation.

Cole, M., Frankel, F., \& Sharp, D. (1971). Development of free recall learning in children. Developmental Psychology, 4, 109-123.

Colom, R., Shin, P. C., Flores-Mendoza, C., \& Quiroga, M. A. (2006). The real relationship between short-term memory and working memory. Memory, 14, 804-813.

CowAn, N. (2001). The magical number 4 in short-term memory: A reconsideration of mental storage capacity. Behavioral \& Brain Sciences, 24, 87-114.

Cowan, N., Nugent, L. D., Elliott, E. M., Ponomarev, I., \& Saults, J. S. (1999). The role of attention in the development of short-term memory: Age differences in the verbal span of apprehension. Child Development, 70, 1082-1097.

Cowan, N., Nugent, L. D., Elliott, E. M., \& Saults, J. S. (2000). Persistence of memory for ignored lists of digits: Areas of developmental constancy and change. Journal of Experimental Child Psychology, 76, 151-172.

Engle, R. W., Tuholski, S. W., Laughlin, J. E., \& Conway, A. R. A. (1999). Working memory, short-term memory, and general fluid intelligence: A latent-variable approach. Journal of Experimental Psychology: General, 128, 309-331.
KAIL, R. V. (2007). Longitudinal evidence that increases in processing speed and working memory enhance children's reasoning. Psychological Science, 18, 312-313.

Kane, M. J., \& ENGLE, R. W. (2002). The role of prefrontal cortex in working memory capacity, executive attention, and general fluid intelligence: An individual-differences perspective. Psychonomic Bulletin \& Review, 9, 637-671.

McGrew, K. S., \& Flanagan, D. P. (1998). The intelligence test desk reference (ITDR): Gf-Gc cross battery assessment. Boston: Allyn \& Bacon.

Perantie, D. C., Wu, J., Koller, J. M., Lim, A., Warren, S. L., Black, K. J., ET AL. (2007). Regional brain volume differences associated with hyperglycemia and severe hypoglycemia in youth with Type 1 diabetes. Diabetes Care, 30, 2331-2337.

Schatz, J., \& Roberts, C. W. (2005). Short-term memory in children with sickle cell disease: Executive versus modality-specific processing deficits. Archives of Clinical Neuropsychology, 20, 1073-1085.

Schrank, F. A., McGrew, K. S., \& Woodcock, R. W. (2001). Assessment Service Bulletin Number 2: WJ III technical abstract. Itasca, IL: Riverside.

Spitzer, T. M. (1976). The development of visual and auditory recall as a function of presentation and probe modalities, serial position and series size. Child Development, 47, 767-778.

Swanson, H. L., \& Howell, M. (2001). Working memory, short-term memory, and speech rate as predictors of children's reading performance at different ages. Journal of Educational Psychology, 93 720-734.

Talmi, D., Grady, C. L., Goshen-Gottstein, Y., \& Moscovitch, M (2005). Neuroimaging the serial position curve: A test of single-store versus dual store models. Psychological Science, 16, 716-723.

TAN, L., \& WARD, G. (2000). A recency-based account of the primacy effect in free recall. Journal of Experimental Psychology: Learning, Memory, \& Cognition, 26, 1589-1625.

Tulving, E., \& Colotla, V. A. (1970). Free recall of trilingual lists. Cognitive Psychology, 1, 86-98.

Unsworth, N., \& ENGLE, R. W. (2007). On the division of short-term and working memory: An examination of simple and complex span and their relation to higher-order abilities. Psychological Bulletin, 133, 1038-1066.

Waugh, N. C., \& Norman, D. A. (1965). Primary memory. Psychological Review, 72, 89-104.

Woodcock, R. W., McGrew, K. S., \& MATHER, N. (2001). WoodcockJohnson III tests of cognitive abilities. Itasca, IL: Riverside.

(Manuscript received May 14, 2008; revision accepted for publication May 7, 2009.) 